\title{
Révision des Neomelaniconion Newstead (Diptera : Culicidae) de Madagascar : espèces présentes et description de cinq nouvelles espèces
}

\author{
Gilbert Le Goff $^{(1)}$, Philippe Boussès ${ }^{(1)} \&$ Jacques Brunhes ${ }^{(2)}$ \\ (1) IRD, UR 016, "Caractérisation et contrôle des populations de vecteurs », BP 64501, F-34394, Montpellier Cedex 5, France
}

(2) 79 rue Pasteur, F-63170, Aubière, France

\begin{abstract}
Revision of the Neomelaniconion Newstead (Diptera: Culicidae) form Madagascar: recorded species and decription of five new species. The authors carry out a review of the genus Neomelaniconion Newstead in Madagascar. They confirm the presence of Neomelaniconion circumluteolus (Theobald 1908). They question the presence of Neomelaniconion palpalis (Newstead 1907) and they describe five new species for world fauna: Neomelaniconion albiradius sp. nov., Neomelaniconion bellecisp. nov., Neomelaniconion fontenilleisp. nov., Neomelaniconion nigropterum sp. nov., Neomelaniconion sylvaticum sp. nov. They finally propose the creation of Circumluteolus and Sylvaticum groups.
\end{abstract}

Résumé. Les auteurs effectuent une révision du genre Neomelaniconion Newstead à Madagascar. Ils confirment la présence de Neomelaniconion circumluteolus (Theobald 1908), mettent en doute la présence de Neomelaniconion palpalis (Newstead 1907) et décrivent 5 nouvelles espèces pour la faune mondiale : Neomelaniconion albiradius sp. nov., Neomelaniconion belleci sp. nov., Neomelaniconion fontenillei sp. nov., Neomelaniconion nigropterum sp. nov., Neomelaniconion sylvaticum sp. nov. Ils proposent enfin la création des groupes Circumluteolus et Sylvaticum.

Keyword: Taxonomy, Culicidae, Neomelaniconion, new species, Madagascar.

$\mathrm{D}$ ans la littérature qui se rapporte aux moustiques de la sous-région zoogéographique malgache, seule la présence de deux espèces appartenant au genre Neomelaniconion a été jusqu'à présent mentionnée. Il s'agit de Neomelaniconion palpalis (Newstead 1907) et de Neomelaniconion circumluteolus (Theobald 1908) ; cette dernière espèce est présente à Madagascar et à Mayotte (Archipel des Comores).

$\mathrm{Ne}$. circumluteolus a été capturé pour la première fois par Hamon en 1959 (com. pers.) puis par Brunhes en 1967. Sa présence était ainsi signalée à Morondava, Manja, Arivonimamo et Ambohimahasoa. Un peu plus tard, cet Aedini a été capturé à Mayotte (Archipel des Comores) par Brunhes (1977) et à Tsiroanomandidy par Ravaonjanahary (1978). Dans son étude sur les circuits de vection d'arbovirus, Fontenille (1989) a signalé sa présence à Madagascar depuis les zones côtières jusqu'aux Hautes Terres centrales et du Nord de l'Île jusqu'au $22^{\text {ème }}$ parallèle de latitude Sud.

La première mention de $N e$. palpalis a été faite par Doucet (1951) qui souligne son importante agressivité diurne dans la forêt primaire de Périnet/Andasibe. Comme nous le verrons dans la discussion, cette mention peut être remise en question.

E-mail: legoff@ird.fr, bousses@ird.fr, jacques.brunhes@free.fr Accepté le 16 novembre 2006
Au cours de son étude effectuée dans plusieurs régions de l'île, Fontenille (op. cit.) a capturé de nombreux Neomelaniconion anthropophiles. En cherchant à identifier ces femelles il a constaté que les clés pour identifier les espèces africaines établies par Le Berre et Hamon (1960), puis par McIntosh (1971), n'étaient pas efficientes à Madagascar. Il apparaissait ainsi clairement qu'une étude préalable d'alphataxonomie était nécessaire avant toute étude sur la transmission des virus.

\section{Historique du genre}

Sous-genre Neomelaniconion Newstead, in Newstead, Dutton and Todd 1907: 31. Haplotype: palpalis Newstead.

Sous-genre Banksinella, Theobald 1907: 469. Haplotype: Culex luteolateralis Theobald.

Genre Neomelaniconion: Reinert, Harbach \& Kitching 2004.

Newstead et al. ont inventé le sous-genre Neomelaniconion le $1^{\text {er }}$ février 1907 en décrivant Aedes (Neomelaniconion) palpalis qui provenait du Zaïre. Pendant plusieurs dizaines d'années la description de ce sous-genre a été attribuée à tort à Theobald qui a décrit, le 23 février 1907, un nouveau taxon provenant de Gambie. Theobald a fait de ce taxon un genre nouveau qu'il a nommé Banksinella luteolateralis var. albothorax. Ce nouveau genre a été rétrogradé plus tard au rang de sous-genre avec le nom d'Aedes (Banksinella) albothorax (Theobald). La courte préséance de Newstead et al. sur celle de Theobald sera reconnue et le sous-genre 
Banksinella a été mis alors en synonymie avec le sousgenre Neomelaniconion. Ce taxon a été dénommé Aedes (Neomelaniconion) albothorax (Theobald 1908) jusqu'à ce que Reinert et al. (2004) élèvent le sous-genre Neomelaniconion au rang de genre et qu'il devienne alors Neomelaniconion albothorax (Theobald 1908).

En 1985, lors de la description d'Aedes (Neomelaniconion) mcintoshi, Huang 1985 attribuait aux membres de ce qui était encore le sous-genre Neomelaniconion les caractères communs suivants: écailles couchées du vertex étroites; écailles fourchues dressées nombreuses sur l'ensemble du vertex; palpes maxillaires du mâle plus longs que le proboscis et formés seulement de 4 segments, palpomère IV redressé et pourvu ventralement de nombreuses et longues soies; soies acrosticales et dorsocentrales présentes; paratergites nus; scutellum portant seulement des écailles étroites.

A ces caractères s'ajoute la présence plus ou moins nette d'une bande latérale d'écailles claires (jaunes ou blanchâtres) qui borde tout le scutum depuis le promontoire jusqu'au scutellum. Cette particularité ornementale permet souvent de reconnaître au premier coup d'œil un imago appartenant à ce genre.

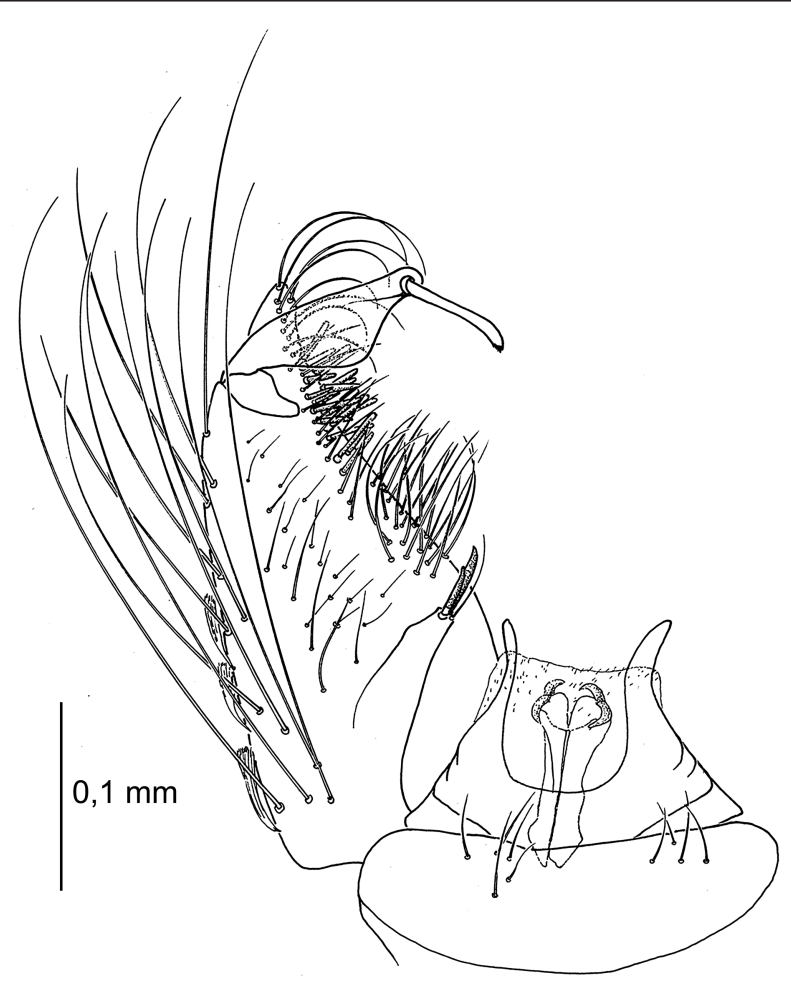

Figure 1

Genitalia de Neomelaniconion circumluteolus Theobald.

\section{Matériel et méthodes}

Pour effectuer cette révision du genre nous avons recherché les Neomelaniconion dans les collections de Doucet, Grjebine, Hamon, Fontenille et de l'un de nous (Brunhes); ces collections sont déposées au Centre IRD de Montpellier (France). Les informations concernant la biologie des espèces malgaches proviennent des carnets de récoltes de Brunhes déposés à Montpellier et non publiés ainsi que des travaux de Fontenille (1989).

Malgré les bouleversements considérables qu'apporte la classification des Aedini proposée par Reinert, Harbach et Kitching (2004) nous l'avons adoptée ici car elle a, à nos yeux, l'avantage de fractionner le très populeux et très hétérogène genre Aedes en plusieurs genres plus homogènes. Nous ne rentrerons pas dans le débat qui oppose actuellement partisans et adversaires de cette classification dont certaines subdivisions sont probablement excessives ou tout au moins insuffisamment démontrées.

Les auteurs ont adopté la terminologie proposée par Harbach et Knight (1980) qui fait l'unanimité chez les spécialistes des moustiques. Dans les descriptions d'imagos, lors de la première mention d'une soie, d'une plaque ou d'un site, nous avons mentionné le développé du nom ainsi que son abréviation. Lors des mentions suivantes nous n'avons très généralement utilisé que l'abréviation. Il nous est cependant arrivé de mentionner le nom complet s'il est placé en début de phrase ou s'il est court et nettement plus explicite que l'abréviation.

Les dessins ont été faits à l'aide d'une chambre claire associée à un microscope Leitz Laborlux D.

Dans la présentation du matériel entomologique étudié nous avons mentionné le nom du récolteur entre parenthèses et en italique.

Dans la collection de Fontenille, la plupart des spécimens porte une abréviation "MD » qui indique que ceux-ci proviennent de Madagascar ; le nombre qui suit cette mention est propre au spécimen concerné ; le nombre qui suit le point indique l'année de la récolte. Ces mentions de Fontenille sont donc du type: MD623.83.

Dans la collection de Brunhes, les spécimens issus d'élevages de larves ou de nymphes portent la lettre «E » comme " élevage "; le nombre qui suit désigne un élevage particulier dont les caractéristiques sont mentionnées dans ses cahiers de récoltes. Les exemplaires qui ne portent pas cette lettre " $\mathrm{E}$ " ont été capturés au filet à main ou sur homme. Les larves qui n'ont pas été placées en élevage portent la lettre " $G$ ».

Le matériel entomologique de référence a été déposé à l'Institut de recherche pour le développement de Montpellier (IRD), au Muséum national d'Histoire naturelle (MNHN), au Natural History Museum de Londres (BMNH) et à l'Instituto de Higiene e Medicina Tropical de Lisbonne (IHMT).

\section{Description des Neomelaniconion de Madagascar}

\section{Neomelaniconion circumluteolus (Theobald 1908)}

Theobald $1908: 107$, , , Banksinella luteolateralis var. circumluteola. Loc. type : Transvaal, (BM).

Edwards $1941: 204$, o $^{*}$ et ㅇ.

Hopkins $1952: 208$, larve*.

McIntosh $1971: 326, \jmath^{*}$ et q*$^{*}$. 
Le matériel malgache que nous utilisons pour redonner une brève description de cette espèce provient d'Ambalavao (Province de Fianarantsoa, 1/ II/1969, récolte au filet à main), (Brunhes) et d'Andapa (Province de Diego-Suarez, XI/1983) (Fontenille). Cette description permettra aux entomologistes malgaches de disposer, sur la même publication, des informations concernant la morphologie de tous les Neomelaniconion présents sur la Grande Île. Nous mettrons particulièrement en évidence les caractères morphologiques qui différencient $N e$. circumluteolus des cinq autres espèces malgaches décrites ci-dessous.

Matériel étudié. Madagascar. Province d'Antananarivo (Tananarive). Ambodimanga (alt. 1000 m) (Fivondronana de Moramanga) : 1 \%, 15/II/1967 (Brunhes). Lac Itasy (alt. $900 \mathrm{~m}$ ) (Îlot Boisé) : 1 q, 1966 (Brunhes). Ivato (village d'Ankodondona) (alt. 1300 m) (Tananarive) : 1 q, 16/I/1987 (Fontenille), agressive de nuit à l'intérieur des habitations. La Mandraka (alt. $1100 \mathrm{~m}$ ) : 2 우, 18/II/1984 (Fontenille), piège lumineux type "CDC Light Trap ", 2 \&, 1/XII/1988 (Fontenille), agressives de nuit, 1 Tे (T.1012), XII/1988 (Fontenille), piège lumineux. Marovitsika (alt. 1000 m) (Fivondronana de Moramanga) : 3 우, I/1988 (Fontenille), 1 ․, 3/III/1988 (Fontenille), agressive en forêt (Lavatrandraka) l'après midi. Tsiroanomandidy (village de Tsaramonenana) (alt. $700 \mathrm{~m}$ ) : 1 + , 16/XII/1985 (Fontenille) piège lumineux. Province d'Antsiranana (Diego-Suarez). Andapa (alt. $500 \mathrm{~m}): 2$ 우, 22/XI/1983 (Fontenille), agressives pour l'homme, 3 ऊิ่ (MD615.83, MD616.83, MD623.83), 26/XI/1983 (Fontenille), 1 9, 27/XI/1983 (Fontenille), agressive pour l'homme. Nosy Be (forêt CNRO) (alt. 10 m) : 1 9, 20/XII/1984 (Fontenille), agressive pour l'homme, 2 오, 21/XII/1984 (Fontenille), agressives pour l'homme. Province de Fianarantsoa. Ambalavao (alt. $900 \mathrm{~m}$ ) : 3 우 et 2 ठิ ô (T.1004, T.1008), 7 /II/1969 (Brunhes). Province de Mahajanga (Majunga). Ankarafantsika-Ampijoroa (alt. 150 m) : 1 f, 17/XII/1982 (Fontenille), agressive pour l'homme (10H00), 1 \%, 12/II/1983 (Fontenille), agressive pour l'homme (16H00), 1 \% , 15/II/1987 (Fontenille), agressive pour l'homme (12H00). Maevatanana (alt. $200 \mathrm{~m}$ ) : 1 q, 19/II/1987 (Fontenille), agressive pour l'homme (18H00). Mampikony (alt. $300 \mathrm{~m}$ ) : 2 우, II/1969 (Brunhes). Marovoay (alt. 100 m) : 4 o 9, II/1969 (Brunhes). Soalala (alt. $10 \mathrm{~m}): 1$ \%, 22/ X/1988 (Fontenille). Province de Toamasina (Tamatave). Ivoloina (alt. $50 \mathrm{~m}$ ) (proche de Tamatave) : 2 우, 27/V/1983 (Fontenille), agressives de nuit pour l'homme. Station forestière de Périnet (alt. $900 \mathrm{~m}$ ) : 1 q, 23/II/1984 (Fontenille), agressive pour l'homme (15H00), 1 q, 19/IV/1985 (Fontenille), agressive pour l'homme (15H00), 1 9, II/1986 (Fontenille), 1 + , 9/V/1986 (Fontenille), agressive pour l'homme (15H00), 1 O, 12/VI/1986 (Fontenille), agressive pour l'homme (15H00). Province de Toliara (Tuléar). Manja (alt. $250 \mathrm{~m}$ ) : 2 오, 19/ V/1959 (Hamon). Morondava (alt. $50 \mathrm{~m}): 6$ q, $\mathrm{V} / 1959$ (Hamon), 1 o, 13/III/1985 (Fontenille), agressive pour l'homme le matin, 1 9, 18/III/1985 (Fontenille), agressive pour l'homme de jour, 1 q, 21/III/1985 (Fontenille), agressive pour l'homme de jour. Archipel des Comores. Mayotte. Dembeni (alt. $20 \mathrm{~m}$ ) : 2 우 agressives de nuit, janvier 1972 (Brunhes).

Redescription de la femelle. Tête. Vertex : orné sur toute sa partie médiane d'une tache blanc jaunâtre bien marquée ; cette tache est encadrée par 2 taches noires ou brun très sombre; présence d'écailles larges et claires sur la partie postgénale; la partie plus ventrale du vertex est à nouveau recouverte d'écailles noires couchées. Tache claire médiane formée d'écailles falciformes plus ou moins dressées, analogues à

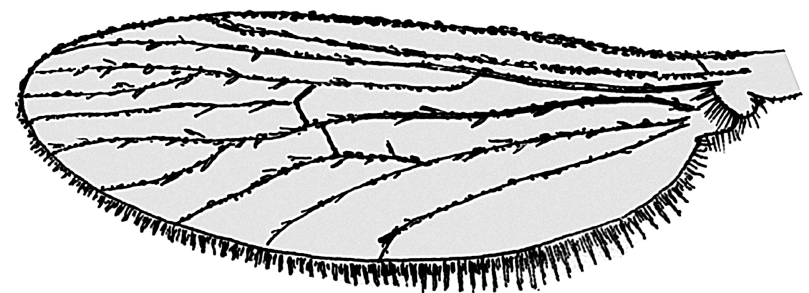

A

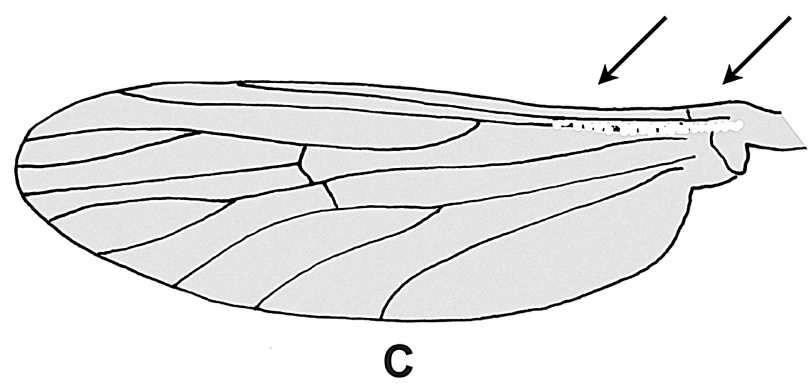

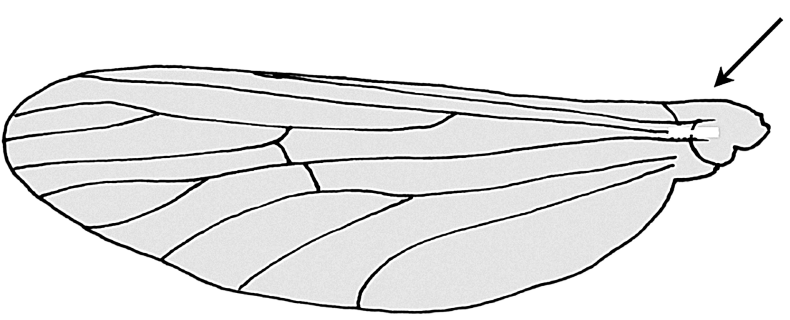

B

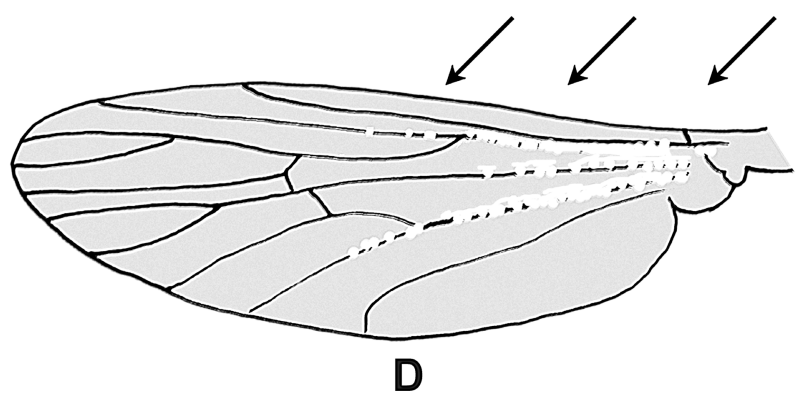

Figure 2

Ailes de femelles. A, Neomelaniconion fontenillei $\mathbf{n}$. sp., Neomelaniconion nigropterum n. sp. et Neomelaniconion sylvaticum $\mathbf{n}$. sp.; B, Neomelaniconion belleci n. sp. ; C, Neomelaniconion albiradius $\mathbf{n}$. sp. ; D, Neomelaniconion circumluteolus. 
celles portées par les côtés du scutum et d'écailles fourchues dressées ; touffe frontale formée de longues écailles claires. Les 2 taches sombres oculaires sont formées d'écailles couchées et de rares écailles fourchues dressées. Antenne. Pédicelle marron clair, plus sombre sur la partie médiane ; premier flagellomère jaunâtre à la base devenant progressivement noir ; les autres flagellomères sont noirs. Proboscis sombre, un peu plus clair dans sa partie médiane. Palpe maxillaire noir. Thorax. Tégument marron foncé. Scutum orné latéralement de 2 larges bandes longitudinales d'écailles jaune d'or qui sont à l'origine du derivatio nominis, de 2 bandes étroites d'écailles jaunes qui suivent intérieurement la ligne des soies dorso-centrales ; aire préscutellaire $(\operatorname{Pr} A)$ médiane nue mais largement recouverte latéralement d'écailles jaunes. Entre ces différentes bandes longitudinales, le scutum est recouvert d'écailles étroites marron clair. Dans la partie antérieure du scutum, les soies fossales antérieures (ASFS) et dorso-centrales antérieures (ADS) sont bien développées. Dans la partie postérieure du scutum, les soies supra-alaires $(\mathrm{SaS})$, préscutellaires $(\mathrm{Prs} S)$ et scutellaires médianes (MSS) sont longues et fortes; elles donnent à cette partie du thorax un aspect très velu. Soies acrosticales absentes ou très rares. Scutellum. les 3 lobes portent de nombreuses soies longues et fortes; le lobe médian est couvert d'écailles étroites jaune clair. Côté du thorax. tégument marron, plus clair que celui du scutum. Antépronotum (Ap) ne portant que de nombreuses soies sombres. Présence de taches d'écailles larges et blanches sur le proépisternum ( $\mathrm{PeSu})$, l'aire sousspiraculaire (SA), le mékatépisternum supérieur et inférieur (MkSU et MkSL) et la partie centrale du mésanépiméron (Mam). Ces différentes taches blanches sont grossièrement alignées et forment une bande blanche latérale. Trois soies post-spiraculaires (PS). Soie mésanépimérale inférieure (MeSL) absente. Aile. écailles sombres implantées sur la plupart des nervures; nervure cubitale $(\mathrm{Cu})$ presque entièrement recouverte d'écailles blanches, seule une partie variable de son apex est sombre; nervure radiale blanche depuis l'insertion de l'aile jusqu'au départ de Rs; quelques écailles claires peuvent être observées sur R1 et Sc; une frange de longues soies sur le calyptère supérieur (UC). Haltère. pédicelle clair et large à la base ; capitellum brun sombre. Pattes. Coxae brun clair ; de nombreuses écailles claires sur la face antérieure de la coxa I ; quelques écailles claires latérales sur les coxae II et III. Pattes entièrement sombres à l'exception des fémurs. Fémur I sombre, seule la partie basale est claire. Fémur II : partie basale et ventrale claire. Patte III : sur la partie ventrale, une longue tache blanche s'étend de la base jusqu'au milieu du segment. Abdomen. Tégument des tergites sombre. Sur le segment I, présence d'une large tache médiane d'écailles claires. Sur le tergite II, présence d'une tache triangulaire blanche dont la base fait suite à la tache portée par le tergite I. Sur les tergites III à VI, présence d'une bande claire basale qui devient de plus en plus étroite de la base vers l'apex de l'abdomen. Ces taches ressemblent à des bandes blanches complètes mais il n'en est rien car une zone sombre les sépare des taches blanches qui recouvrent les angles antérieurs des tergites III à VI. Tergite VII avec 2 bandes blanches; la bande blanche distale est nettement plus étroite que la bande basale. Sternites I à VI partiellement couverts d'écailles claires, les apex des sternites V à VII sont moins densément couverts d'écailles.

Redescription du mâle (fig. 1). Ressemble à la femelle mais les antennes présentent de très nombreux verticilles roux. Palpe maxillaire plus long que le proboscis et nettement velu à l'apex ; de nombreuses rangées de longues soies sont présentes sur le quart distal du troisième et quatrième article palpal. Aile. Les écailles claires sont peu nombreuses sur $\mathrm{R}$ et pratiquement absentes sur $\mathrm{R} 1$; sur $\mathrm{Cu}$, elles s'étendent peu vers l'apex. Abdomen. Sombre et pourvu de nombreuses soies longues et noires. Sur les tergites II à VI, présence d'une bande claire basale qui n'atteint pas les angles antérieurs; triangles clairs latéroantérieurs absents. Sternites beaucoup plus sombres que chez la femelle. Genitalia. Sternite IX : 4 à 6 soies fines de chaque côté ; elles sont moins développées que celles de $\mathrm{Ne}$. belleci. L'édéage présente 3 longs crochets recourbés. Paraproctes longs, droits et arrondis à l'apex. Gonocoxite environ 4 fois plus long que large ; sur sa face dorsale sont implantées des écailles et de nombreuses soies longues et arquées. Triangle distal une fois et demi plus long que large; il porte une vingtaine de soies longues, souples et presque bouclées; soies distales plus longues que les soies basales. Une vingtaine d'épines épaisses, courtes et sombres, sont implantées au niveau de l'articulation du gonostyle. Epines distales plus courtes que les épines basales. Présence de soies longues et fines sur toute la longueur du bord interne du gonocoxite. Sur la partie basale, les soies sont nombreuses et forment une touffe de 25 à 30 soies peu serrées. Lobe parabasal portant 2 fortes baguettes légèrement crochues à l'apex ainsi qu'une fine soie. Gonostyle : renflé dans sa partie ventrale; absence de très petites soies sur sa partie dorsale. Griffe du gonostyle légèrement recourbée et terminée par une petite membrane frangée.

Répartition et biologie. Pour esquisser la carte de répartition de Ne. circumluteolus à Madagascar nous n'avons pris en compte que les occurrences que nous avons pu vérifier au cours de cette révision. Utiliser les mentions faites dans la littérature nous aurait fait courir le risque de mêler aux $N e$. circumluteolus des espèces mal identifiées.

Dans la sous-région malgache, Ne. circumluteolus semble largement distribué; on le rencontre dans l'Archipel des Comores (Mayotte) et à Madagascar. Sur la Grande Ile, l'espèce est présente dans les régions côtières de l'Ouest (Morondava, Nosy Be) ou de l'Est (Ivoloina) ainsi que sur les Hautes Terres (Andapa, La Mandraka, Tsiroanomandidy, Ambalavao). A noter toutefois qu'il n'a jamais été fait mention de cette espèce dans le domaine bioclimatique semi-aride du sud-ouest de Madagascar.

L'essentiel des informations dont nous disposons actuellement sur la biologie de $N e$. circumluteolus à Madagascar ont été obtenues par Fontenille (1989). L'espèce est agressive pour l'homme, tout particulièrement pendant la journée et sous couvert forestier. Elle a été capturée sur homme jusqu'au milieu de la nuit, mais en densité plus faible que pendant la journée. Quand les densités étaient élevées (Ampijoroa, Anjiro), l'agressivité diurne a été 3,5 à 4 fois plus élevée que l'agressivité nocturne. A Andapa, en novembre 1983, l'agressivité diurne de cette espèce a été exceptionnelle, jusqu'à représenter près de 59\% de l'ensemble des captures de moustiques $(n=2416)$.

Des tentatives de capture sur lémuriens à Ampijoroa, Andapa et Périnet ont été infructueuses. Par ailleurs, cette espèce a montré un tropisme positif mais modéré pour la lumière. 
La participation de cette espèce à la transmission -ou la dissémination- du virus West Nile à Madagascar (Ampijoroa, décembre 1982) a été suspectée. Cette hypothèse n'a jamais été confirmée depuis cette date.

\section{Neomelaniconion palpale Newstead 1907}

Newstead $1907: 31$, ¡* $^{*}$, Neomelaniconion palpalis

Loc. type : Basoko, Congo Belge (République Démocratique du Congo) (BM).

Edwards $1941: 209$, j* $^{*}$ et ㅇ.

Hopkins $1952: 211$, larve*.

McIntosh $1971: 331$, ठै* $^{*}$ et ..

Reinert et al. 2004 : 360, Neomelaniconion palpale

Doucet (1951) a signalé la présence de Ne. palpale dans la région orientale de Madagascar (Périnet/ Andasibe). Nous pensons que cette présence est à remettre en cause. En effet, Doucet a identifié Ne. palpale en se fondant sur des femelles agressives et sans rechercher, et à plus forte raison disséquer, les mâles. Or, dès que les femelles ne présentaient pas de bandes blanches sur les tergites abdominaux, la clé d'identification qu'il utilisait, et qui était la seule disponible à l'époque (Edwards 1941), le conduisait immanquablement vers "Aedes(Banksinella) palpalis". Par ailleurs, les larves de Ne. palpale, qui se distinguent aisément de celles du Groupe Circumluteolus, n'ont jamais été récoltées à Madagascar. Nous avons examiné des femelles agressives récoltées à Périnet/Andasibe par Doucet ; elles sont effectivement dépourvues de bandes basales claires sur les tergites, par ailleurs leurs ailes ne portent que des écailles sombres et elles appartiennent à $N e$. nigropterum n. sp., à Ne. fontenillei. n. sp. ou à Ne. sylvaticum n. sp. En conséquence, et en attendant la confirmation de la présence de $N e$. palpale qui serait fondée sur l'identification de mâles ou de larves, nous considérons qu'il s'agit d'une erreur d'identification due à l'absence de clés adaptées et à la trop faible connaissance des Culicidae malgaches.

\section{Neomelaniconion belleci $\mathrm{n} . \mathrm{sp}$.}

Matériel type. La série type a été récoltée le 6/IV/1967, à 10 $\mathrm{km}$ au-dessus de la station thermale de Ranomafana [21 $06^{\prime} \mathrm{S}$ $47^{\circ} 21^{\prime} \mathrm{E}$ ] (Province de Fianarantsoa), à une altitude voisine de $1000 \mathrm{~m}$ (Brunhes). Les larves se développaient dans une mare temporaire située sous couvert forestier; l'eau du gîte était légèrement brunâtre et les feuilles mortes y étaient nombreuses. Dans le même gîte se développaient Culex (Neoculex) chauveti Brunhes \& Rambelo 1968 et Anopheles (Anopheles) coustani Laveran 1900. Les larves prélevées ont été mises en élevage (E114) ; elles nous ont permis d'obtenir les nymphes et les adultes de cette nouvelle espèce. Un nouveau prélèvement effectué dans le même gîte quelques jours après (28 avril 1967), nous a montré que $C x$. $(N c x$.) chauveti et $A n$. (Ano.) coustani étaient toujours présents et qu'Anopheles (Cellia) squamosus Theobald 1901 avait, depuis le premier passage, colonisé le gîte ; les Neomelaniconion étaient par contre absents.
Le 25 novembre 1967, c'est à dire au début de la saison des pluies de la même année, nous avons revisité le gîte et constaté que $N$ e. belleci y était à nouveau présent. Les larves récoltées ont été placées en élevage (E210); elles nous ont permis d'obtenir 2

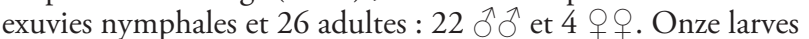
ont été sacrifiées et montées dans l'euparal.

Nous considérons ces 2 élevages comme faisant partie de la série type.

Holotype. 1 đonté sur minutie dont les génitalias portent les $\mathrm{n}^{\circ}$ T.335, E114. Paratypes: la série type comprend 8 larves étiquetées E114, 11 larves étiquetées E210, 2 exuvies

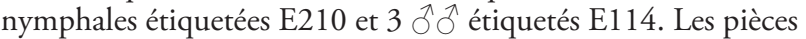
génitales de 2 de ces $\widehat{\partial} \widehat{\partial}$ ont été montées dans l'euparal; ils proviennent de l'élevage E1 14 et portent les n T.333 et T.334. Vingt quatre $\widehat{\partial} \widehat{\jmath}$ (dont T.1002, T.1003, T.1005 et T.1007) et 4 oq proviennent de l'élevage E210. Dépôt des types. L'holotype et les paratypes T.335, T.1003 et T.1005 seront conservés au Centre de faunistique de l'IRD à Montpellier ainsi que $15 \widehat{\jmath} \widehat{\jmath}$ entiers et 2 우. Les 2 exuvies nymphales ayant servi à la description du stade nymphal (E210) seront conservées à l'IRD de Montpellier de même que 9 larves. Deux

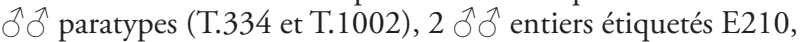
1 O E210, 6 larves étiquetées E114 et 4 larves étiquetées E210

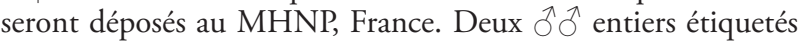

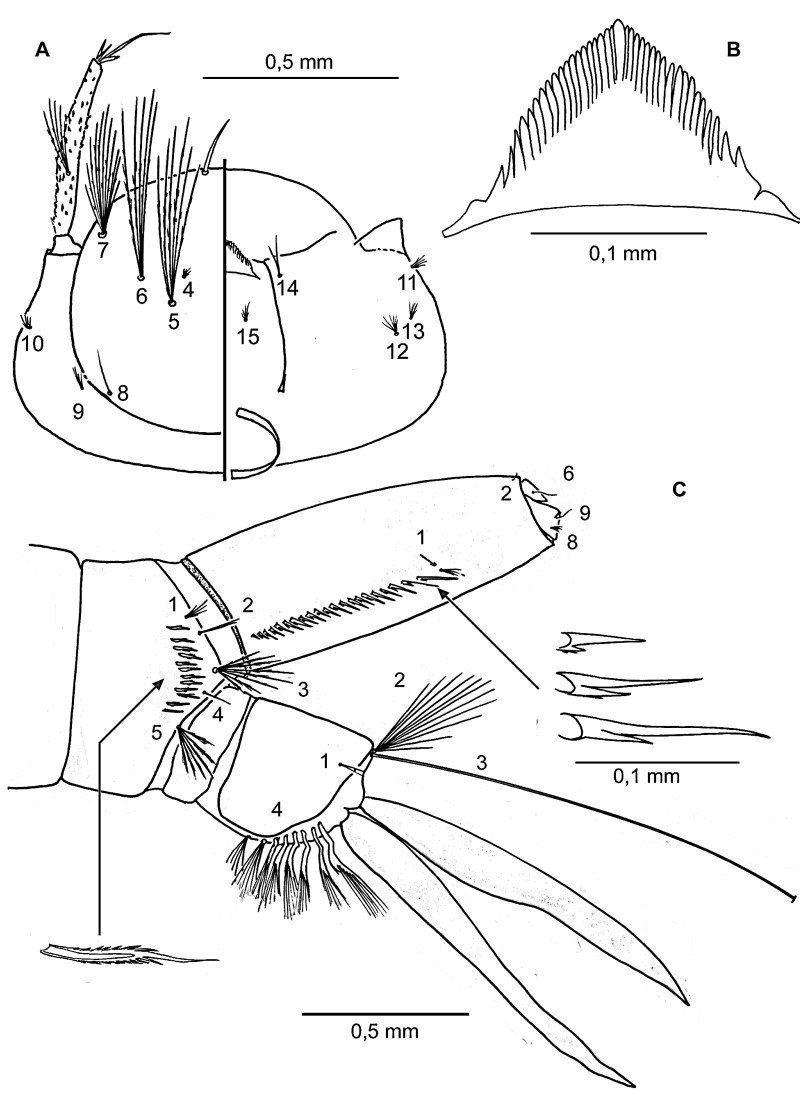

Figure 3

Larve de Neomelaniconion belleci $\mathbf{n}$. sp. A, tête (face dorsale à gauche, face ventrale à droite) ; $\mathrm{B}$, mentum; $\mathrm{C}$, derniers segments de l'abdomen. 
E210, 1 đ dont les génitalias sont montés (T.1005), 2 larves E114 et 4 larves E210 seront déposés au BNMH, Londres.

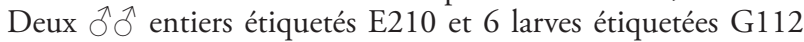
seront déposés à l'IHMT de Lisbonne.

Derivatio nominis. Nom attribué en hommage à Christian Bellec, entomologiste médical de l'IRD, dont le soutien au développement de la taxonomie des insectes hématophages a toujours été fort et constant.

Autre matériel examiné. Province de Fianarantsoa. Ranomafana, G112, même gîte que E114 et E210, III/1969, 30 larves montées dans l'euparal (Brunhes).

Description de l'holotype mâle (fig. 2, 3 et 4). Tête. Vertex. des écailles couchées larges et claires sont présentes sur les côtés du vertex. Sur la partie médiane, autour de la suture coronale (cs) et dans l'espace interoculaire, les écailles couchées sont étroites et jaunâtres. Ces 2 groupes d'écailles claires sont séparés par une zone sombre. Sur l'occiput, les écailles fourchues noires sont présentes mais peu nombreuses. Proboscis entièrement recouvert d'écailles noires, légèrement renflé à l'apex. Palpe maxillaire nettement plus long que le proboscis, recouvert d'écailles sombres ; segment 5 , très petit et triangulaire, portant 2 à 3 soies terminales dressées; segment 4 dressé, portant 2 rangées de soies l'une sur son bord latéral externe et l'autre sur son bord latéral interne; segment 3 pourvu lui aussi de nombreuses soies sur son tiers distal. Antenne. Pédicelle sombre, sans écailles; flagellomères 1 à 12 portant de longs verticilles brun clair. Thorax. Tégument marron foncé sur la partie dorsale devenant plus clair ventralement. Scutum recouvert d'écailles falciformes blanc jaunâtre qui forment un revêtement plus dense sur les côtés. La bordure latérale d'écailles jaunes, qui est si caractéristique du groupe Circumluteolus, est ici présente mais peu marquée. Les écailles, particulièrement abondantes sur le promontoire et les fossae, sont plus claires vers l'insertion des ailes. Des écailles jaunâtres, claisemées et petites, forment deux bandes dorso-centrales mal définies. Aire préscutellaire $(\operatorname{PrA})$ recouverte de soies jaunes falciformes. Soies acrosticales présentes mais peu nombreuses. Soies dorsocentrales postérieures seules présentes. Scutellum avec de nombreuses écailles falciformes jaunâtres couchées: lobe médian avec 7 à 8 soies de taille inégale et disposées en éventail ; lobes latéraux avec 3 à 4 soies longues. Côté du thorax. Paratergite nu (Pa); Ap à tégument sombre sans écailles mais avec de nombreuses soies. Proépisternum (Ps) sans écailles claires. Quatre taches d'écailles blanches sont bien visibles : 2 sont implantées sur le Mks, l'une sur le MScL et l'autre sur le $\mathrm{MScU}$; une importante tache blanche est portée par le Mam, près des soies $\mathrm{MeSU}$. La quatrième tache blanche, plus réduite, se trouve sur la zone SA. Présence de 5 soies post-spiraculaires ainsi que de quelques rares écailles sur PA. Soies mésanépimérales inférieures absentes. Aile. Des écailles sombres implantées sur toutes les nervures. Seule, la base de $\mathrm{R}$, avant l'insertion de la transverse humérale " $\mathrm{h}$ ", apparaît légèrement plus claire. $\mathrm{Al}$ avec une bordure de soies courtes et peu nombreuses; une frange de longues soies sur UC. Haltère. Pédicelle clair et large à la base; capitellum brun sombre. Pattes. Coxae brun clair ; de nombreuses écailles claires sur la face antérieure de la coxa I ; quelques écailles claires sur les coxae II et III. Le reste des pattes est entièrement sombre à l'exception de la base des fémurs. Fémur I sombre, seule la partie basale est claire. Fémur II : partie basale et ventrale claire. Patte III : sur la partie ventrale, une longue tache blanche s'étend de la base jusqu'au milieu du segment. Abdomen. Tégument des tergites brun clair mais présentant des zones plus sombres sur la partie médiane des segments I à IV ainsi qu'à l'apex des tergites IV à VII. Présence de nombreuses et longues soies dorsales et ventrales. Présence de nombreuses écailles claires et sombres mêlées; les écailles sombres sont plus rares que les claires. Sur les segments I et II, le semi d'écailles claires est plus dense en position médiane. Sur les segments III à $\mathrm{V}$, les écailles claires se concentrent sur la partie

Figure 4

Nymphe de Neomelaniconion belleci $\mathbf{n}$. sp. A, céphalothorax; B, métathorax et abdomen (face dorsale à gauche, face ventrale à droite). Mâle. C, genitalia. 
basale du tergite. Sur les tergites VI et VII, les écailles claires forment une bande basale claire. Le contraste, que l'on observe généralement dans le groupe Circumluteolus entre les tergites sombres sur lesquels tranche une bande basale claire, n' existe ici que sur les tergites VI et VII. Tergite VIII recouvert d'écailles blanches. Sternites I à VII recouverts d'écailles claires. Sternite VIII sombre mais piqueté d'écailles claires. Genitalia (Fig. 4). Sternite IX avec de 5 à 6 longues soies fines (rarement 7), de taille inégale, dont les plus longues atteignent l'apex de l'édéage. L'édéage présente 3 longs crochets recourbés, presque semicirculaires. Paraproctes longs, légèrement divergents et arrondis à l'apex. Gonocoxite 4 fois plus long que large ; présence, sur sa face dorsale, d'écailles et de nombreuses soies qui sont d'autant plus longues qu'elles sont plus apicales. Triangle apical à peine plus long que large avec de 10 à 15 soies de tailles inégales; les soies distales sont plus longues que les soies basales. De 10 à 15 épines épaisses, courtes et sombres s'insèrent au niveau de l'articulation du gonostyle. Un peu plus vers la base du gonocoxite se trouvent de 4 à 8 épines plus longues et plus claires que les précédentes. Une touffe lâche de longues soies fines (15 environ) est implantée dans la partie médiane et interne du gonocoxite. Le lobe parabasal porte 2 fortes baguettes droites et 1 soie fine. Gonostyle renflé dans sa partie ventrale; présence de spicules épars, plus nombreux sur la partie dorso-apicale ; une fine membrane dorsale bordée d'épines se développe à l'apex du gonostyle, elle peut se replier et devient alors peu visible ; épine terminale légèrement recourbée et terminée par une petite membrane frangée.

Femelle (fig. 2-B). Morphologie très voisine de celle du mâle. L'ornementation d'écailles claires des pleures et de l'abdomen est plus fournie que chez le mâle. Une importante plage d'écailles claires est présente sur les tergites I et II. Sur les tergites III à VI, les écailles claires forment une bande occupant le tiers basal du segment; sur les tergites V et VI, ces bandes claires s'élargissent latéralement. La partie distale des tergites II à VI est recouverte d'écailles sombres. Sur le tergite VII, les écailles claires forment une bande basale plus étroite ainsi que 2 taches latérales qui occupent toute la longueur du segment. Comme chez le mâle, les sternites I à VI sont recouverts d'écailles claires (particulièrement denses sur les segment V et VI) ; sternite VII recouvert d'un mélange d'écailles claires et sombres. Cerque noire.

Nymphe (fig. 4). Tégument clair et sans marques particulières. La très grande majorité des soies du céphalothorax et de l'abdomen sont petites, claires et donc peu visibles. Cephalothorax. Trompette respiratoire cylindrique, environ 3 fois plus longue que large; la partie distale du meatus est nettement plus spiculée que les $2 / 3$ basaux. Soies céphalothoraciques. 1-CT: 3 branches; 2-CT : 3 à 4 branches; 3-CT : 1 à 2 branches; 4-CT: une hampe basale fourchue à l'apex; 5-CT: 8 à 10 branches souples; 6-CT : 1 hampe subdivisée en 3 ou 4 branches apicales; 7-CT : 2 branches fourchues à l'apex ; 8CT : 7 à 8 branches fines et souples; 9-CT : 2 à 3 branches fines; 10-CT : 4 branches très courtes; 11-CT : 2 branches relativement longues; 12-CT: 1 longue hampe fourchue. Abdomen. Soies abdominales. 1-I : 10 à 15 branches simples et souples. Seules les soies 6-I, 6-II, 1-III, 5-IV, 10-V et 7VI sont longues et relativement bien visibles. Les autres soies abdominales sont courtes, fines et presque transparentes. Palette natatoire : bord distal externe et bord interne clairs, peu visibles; nervure médiane nettement marquée à la base mais n'atteignant pas l'apex. Soie 1-P à 2 branches courtes.
Larve (fig. 3). Aspect général : corps jaunâtre; assez grande taille (plus de $5 \mathrm{~mm}$ ); capsule céphalique, siphon et segment anal brunâtres. Tête. Antenne cylindrique, plus longue que la moitié de la longueur de la tête, présence de nombreux spicules sur tout le tégument; sur la partie basale et externe ces spicules sont forts et denses ; soie 1-A insérée un peu en deçà de la moitié de la longueur de l'antenne; formée de 5-7 branches finement aciculées à l'apex. Soies céphaliques. Soie $4-\mathrm{C}: 5$ à 7 branches courtes et souples ; $5-\mathrm{C}, 6-\mathrm{C}$ et $7-\mathrm{C}$ avec respectivement $5-6$, 5 et $8-10$ branches aciculées sur toute leur longueur ; 8 -C et 9-C : 3 et 4-5 branches courtes, fines et souples ; 14-C longue et bifide. Mentum triangulaire, 15-17 dents latérales serrées les unes contre les autres, dent centrale forte; dents latérales nettement séparées. Thorax. Prothorax. Soie 0-P : petite touffe de 20-25 branches courtes et souples ; 1-P, 2-P, 3-P et 4-P formées respectivement de 2-3, 2-3, 3-5 et 3-4 branches courtes et souples ; 5-P, 6-P, 7-P et 8-P avec respectivement, 1 , 1,3 et 3 (rarement 4 ) branches fortes, longues et aciculées sur toute leur longueur. Mésothorax. Soie 5-M : 1 branche épaisse et aciculée ; 6-M : divisée en 6-9 branches aciculées ; 7-M : 1 branche aciculée dans la partie médiane ; 8-M et 9-M en éventail avec respectivement, 5-9 et 6-8 fortes branches aciculées ; 10$\mathrm{M}$ et $12-\mathrm{M}$, chacune avec une longue branche droite aciculée sur toute leur longueur. Métathorax. Soie 7-T formée de 7-10 fortes branches aciculées et en éventail ; 9-T et 10-T formées respectivement de $1-3$ et 1 branches longues, fortes et aciculées. Abdomen. Segment I. Soies 6-I et 7-I : respectivement 2-3 branches et 1-2 branches fortes et aciculées. Segments II à VI. Soie 6-II : 2-3 branches fortes et aciculées ; 6-III à 6-VI : 1 branche aciculée. Segment VIII. Peigne formé d'une douzaine de dents disposées grossièrement en arc de cercle; ces dents présentent une épine médiane longue et acérée et de fines denticulations latérales ; 1-VIII : 3-5 branches ; 2-VIII bifide ; 3-VIII : une dizaine de branches droites et aciculées ; 4-VIII bifide ou simple; 5-VIII : 6-8 branches droites et finement aciculées. Siphon sombre; index compris entre 2,2 et 2,9 (moyenne : 2,5) ; peigne ne dépassant pas l'insertion de la soie $1-S$, et composé de 20 à 21 dents de tailles croissantes de la base vers l'apex. Ces dents portent toujours un fort denticule basal auquel s'ajoutent parfois de petits denticules. Dents apicales plus développées et plus espacées que les dents basales. Soie 1$S$ ayant la forme d'une petite touffe de 3-5 branches fines et souples. Segment X. Selle presque complète et non armée ; soie 1 -X courte et très généralement bifide ; $2-X$ : 9-12 branches en éventail ; 3-X : très longue et simple; 4-X: 6 paires de soies subdivisées en $8-12$ branches et de 2 soies impaires formées de 6-10 branches lisses et courtes. Papilles anales 2 à 3 fois plus longues que la selle, étroites et légèrement lancéolées.

Biologie et répartition. Les larves de $\mathrm{Ne}$. belleci n. sp. ont été capturées alors qu'elles se développaient dans une mare temporaire située sous couvert forestier. La matière organique en décomposition (bois et feuilles) était abondante et l'eau était riche en tannin. Ce type de gîte temporaire dans lequel apparaît, dès la première remise en eau, une population larvaire est conforme à ceux décrits par Hopkins (1952) pour Ne. circumluteolus. Cependant, la présence dans le même gîte de larves de $N e$. belleci n. sp. en fin de saison des pluies (mars et avril) montre que plusieurs générations peuvent s'y développer chaque année en fonction du 
rythme des remises en eau. Dans ce gîte $C x$. $(N c x$. $)$ chauveti, An. (Ano.) coustani et An. (Cel.) squamosus étaient présents.

Dans l'état très fragmentaire des connaissances actuelles cette espèce ne semble présente que dans la forêt orientale et à une altitude voisine de $900 \mathrm{~m}$.

\section{Neomelaniconion nigropterum $\mathbf{n}$. sp.}

Matériel type. La série type a été récoltée en février 1984, en avril 1985, en février et mai 1986 et en janvier 1987, dans la station forestière de Périnet/Andasibe [18 $8^{\circ} 55^{\prime} \mathrm{S} 48^{\circ} 25^{\prime} \mathrm{E}$ ] (Province de Toamasina), à une altitude voisine de $900 \mathrm{~m}$ (Fontenille). Les adultes ont été collectés, en sous bois, sur homme ou au filet à main. Les stades préimaginaux sont à ce jour inconnus.

Holotype : Un $\widehat{\jmath}$ monté sur minutie dont les génitalias portent le $\mathrm{n}^{\circ}$ MD42.84, Périnet, février 1984. Paratypes: Station forestière de Périnet/Andasibe (alt. 900 m) : 1 O (MD67.84, identification moléculaire), 24/II/1984 (Fontenille), agressive pour l'homme de jour et $2 \widehat{\jmath} \widehat{\jmath}$ (MD28.84, MD63.84), 2023-24/II/1984 (Fontenille), filet à main. 2 ऊ̂̉ (MD173.85, MD174.85), 19/IV/1985 (Fontenille), filet à main. 1 đ (MD6.86), 6/I/1986 (Fontenille), filet à main. 14 ठึત (MD40.86, MD45.86, MD55.86, MD89.86, MD91.86, MD92.86, MD94.86, MD95.86, MD96.86, MD99.86, MD100.86, MD101.86, MD102.86, MD103.86), 4-5-6 et 28II/1986 (Fontenille), filet à main. 2 우 (MD182.86 et MD209.86, identification moléculaire), 9 et $11 / \mathrm{V} / 1986$ (Fontenille), agressives pour l'homme l'après-midi. 1 त (MD11.87), 23/I/1987 (Fontenille), filet à main. 4 ठึ (T.1000, T.1006, T.1009, T.1010), 12/I/1988 (Fontenille). 3 오우 (MD.82) ; 7 우 MD.83; 8 우으 MD.84; 3 우우 MD.85 et 23 우 MD.86) (Fontenille).

Dépôt des types. L'holotype sera déposé dans la collection d'entomologie médicale de l'IRD, Centre de Montpellier, France. Trois paratypes $\hat{\jmath} \widehat{\partial}$ (MD40.86, MD45.86, MD55.86) et 3 paratypes 우 (MD.83) seront déposés au Muséum national d'Histoire naturelle, Paris, France. Deux paratypes ग̃र (MD89.86, MD91.86) et 2 paratypes 0 우 (étiquetés MD.84) seront déposés au Natural History Museum, Londres, Royaume-Uni.

Derivatio nominis. Nom en rapport avec les ailes entièrement sombres de cette espèce.

Holotype mâle (fig. 4). Tête. Vertex. Tégument brun foncé ; un semis d'écailles couchées étroites et jaunâtres autour de la suture coronale (cs) et dans l'espace interoculaire ; sur la zone oculaire, présence d'une tache d'écailles couchées larges et blanchâtres; ces 2 tâches d'écailles claires sont séparées par une zone sombre sur laquelle les écailles larges et claires sont peu nombreuses. Des écailles fourchues noires sur l'occiput. Proboscis entièrement recouvert d'écailles noires. Palpe maxillaire plus long que le proboscis et recouvert d'écailles sombres; le tiers apical du segment 3, ainsi que le segments 4 , portent ventralement de longues soies disposées en 2 séries divergentes, le segment 5 est triangulaire, il porte quelques soies longues et raides. Antenne. Pédicelle nu et sombre; verticilles des flagellomères 1 à 12 de couleur brun clair ; les flagellomères 13 et 14 sont longs et fins, l'apex du flagellomère 13 porte 4 à 5 longs verticilles. Thorax. Tégument noir. Scutum recouvert d'écailles falciformes jaunâtres sur la partie sagittale; latéralement les écailles sont plus longues, plus larges et blanchâtres, elles forment 2 bandes claires latérales qui ceinturent le scutum jusqu'à l'aire préscutellaire, 2 lignes dorso-centrales mal définies sont tout de même perceptibles. Soies acrosticales et soies dorsocentrales antérieures et postérieures rares. Soies supra-alaires particulièrement longues, fortes et nombreuses. Scutellum. Lobe médian recouvert d'écailles claires couchées et portant de nombreuses soies longues et fortes; les lobes latéraux portent 5 soies dont 3 sont longues et fortes; ils ne présentent que de très rares écailles jaunâtres. Partie latérale du thorax. Pronotum antérieur (Ap) et postérieur (Ppn) dépourvus d'écaille mais portant de nombreuses longues soies. Proépisternum (Ps) portant quelques écailles claires et de nombreuses soies ; 5 soies postspiraculaires ainsi que quelques écailles sur PA. Les écailles sont présentes sur SSc. Présence de seulement 3 taches d'écailles blanchâtres bien visibles. Deux de ces taches se trouvent sur le $\mathrm{Mks}$; la tache $\mathrm{MscU}$ est formée d'écailles couchées alors que la tache MScL est formée d'écailles nettement dressées. La troisième tache, la $\mathrm{AMsC}$, est formée d'écailles couchées. Soie mésanépimérale inférieure absente. Aile. Entièrement recouverte d'écailles sombres ; alula portant une ligne d'écailles sombres ; une frange de longues soies sur UC. Haltère. Pédicelle clair; capitellum brun et glabre. Pattes. Coxae brun clair ; quelques écailles claires sur la face antérieure de la coxa I ; coxae II et III dépourvues d'écailles. Pattes entièrement sombres à l'exception des fémurs ; fémurs I et II sombres antérieurement et clairs sur leur partie postérieure. Le fémur III est blanc à l'exception de sa moitié dorsale et distale. Griffes des pattes I à III simples.

Abdomen. Présence de nombreuses et longues soies marron clair. Tégument des tergites brun couvert d'écailles brun clair et brun sombre qui forment des dessins peu nets. Tergite I : couvert d'écailles brun clair à l'exception d'un tache médiane allongée d'écailles sombres. Tergite II à IV avec une bande médiane souvent peu nette d'écailles brun sombre dont l'apex s'évase pour occuper de plus en plus de place sur le bord du segment. Les parties latérales de ces tergites sont recouvertes d'écailles plus claires. Tergites IV à VII uniformément recouverts d'écailles brun sombre. Sternites II à VII de plus en plus densément recouverts d'écailles larges marron clair de la base vers l'apex ; elles couvrent entièrement les sternites VI et VII. Genitalia. Sternite IX avec de 4 à 7 (généralement 5) soies longues, fines et de tailles inégales. L'édéage présente 3 longs crochets recourbés, presque semi-circulaires. Paraproctes longs, légèrement divergents et arrondis à l'apex. Gonocoxite 5 fois plus long que large ; présence, sur sa face dorsale, d'écailles et de nombreuses et longues soies. Triangle apical à peine plus long que large avec de nombreuses soies implantées aussi bien sur la face dorsale que sur la face ventrale; les soies distales sont plus longues que les soies basales. Une petite dizaine d'épines épaisses, courtes, sombres et serrées les unes contre les autres, est implantée au niveau de l'articulation du gonostyle. Un peu plus vers la base du gonocoxite s'insèrent 4 à 6 épines dont la forme est intermédiaire entre les épines épaisses implantées près du gonostyle et les soies longues et fines qui se trouvent à la base du gonocoxite. Ces soies forment une touffe lâche implantée dans la partie médiane et interne du gonocoxite. Le lobe parabasal porte 1 ou 2 fortes baguettes droites (parfois 1 baguette sur un côté et 2 sur l'autre) et 2 soies fines. Gonostyle renflé dans sa partie ventrale ; présence d'une fine membrane dorsale portant des soies bien visibles; griffe légèrement recourbée et terminée par une petite membrane frangée.

Femelle (fig. 2). Morphologie très voisine de celle du mâle. 
Elle est cependant sensiblement plus ornementée que le mâle et présente les variations suivantes: des écailles blanchâtres $\mathrm{PaSc}$ ainsi que quelques écailles SSc, ApSc et PScU. L'abdomen apparaît noir sur la face dorsale et blanc sur la face ventrale. Les écailles noires recouvrent pratiquement tous les tergites. Une tache d'écailles claires est cependant présente sur l'angle antérieur de chaque tergite, cette tache devient de plus en plus longue de l'avant vers l'arrière jusqu'à occuper la totalité des côtés des tergites VI et VII. Ces taches d'écailles claires ne sont visibles que ventralement. Associées aux sternites couverts d'écailles blanches, ces taches d'écailles claires contribuent à donner un aspect blanc à la face ventrale de l'abdomen des femelles gorgées. Sternites recouverts d'écailles blanchâtres ; les sternites II et VII apparaissent plus sombres car les écailles claires y sont moins nombreuses et le tégument sombre apparaît plus nettement. Cerque noire.

\section{Neomelaniconion sylvaticum n. sp.}

Matériel type. La série type a été récoltée en février 1984, puis en janvier, février et mai 1986 dans la station forestière de Périnet/Andasibe [18 $8^{\circ} 55^{\prime} \mathrm{S} 48^{\circ} 25^{\prime} \mathrm{E}$ ] (Province de Toamasina), à une altitude voisine de $900 \mathrm{~m}$ (Fontenille). Les adultes ont été collectés en sous bois et au filet à main. Les stades préimaginaux sont inconnus.

Holotype. đิ étiqueté MD32.84, Périnet, 21/II/1984. Paratypes : 7 đरे (MD2.86, MD3.86, MD4.86, MD5.86, MD16.86, MD17.86, MD18.86) du 6 et 8/I/1986; 1 ठ $(\mathrm{MD} 46.86) \mathrm{du}$ 5/II/1986; 2 o (MD193.86, MD194.86) du 10/V/1986.

Dépôt des types. L'holotype $\widehat{\sigma}$ ainsi que 5 paratypes seront conservés dans la collection de l'IRD à Montpellier. Trois paratypes seront conservés au Muséum national d'Histoire naturelle de Paris, France et 2 au Natural History Museum, Londres, Royaume-Uni.

Derivatio nominis. Nom en rapport avec l'écologie forestière de cette espèce.

Autre matériel étudié. Province d'Antsiranana (DiegoSuarez). Andapa (alt. 500 m) : 2 đ̋ (MD614.83, MD622.83) 26/XI/1983 ; 5 우 (MD583.83, MD592.83, MD593.83, MD608.83, MD606.83) (Fontenille). Sambava (bord de mer) : 1 q (MD631.83) XI/1983 (Fontenille). Province de Fianarantsoa. Ifaho (alt. $30 \mathrm{~m}): 1 q$ anthropophile, 10/1966 (Brunhes). Manombo (alt. 250 m) (Fivondronana de Farafangana) : 1 ( (MD67.87), 4 우 (MD58.87, MD68.87, MD69.87, MD70.87) III/87 (Fontenille). Province de Toamasina (Tamatave). Fenerive-Est (bord de mer) : 4 우 (06/90), 2 우 (MD122.84, MD123.84) (Fontenille). Ile de Sainte Marie (alt. $10 \mathrm{~m})$ : 2 ऊิ $\widehat{\jmath}$ (MD3.89, MD9.89), 3 우우 (MD1.89, MD2.89, MD8.89) I/1989 (Fontenille); 1 ô (T.1001) 21/V/1989 (Fontenille). Tamatave (bord de mer) : 3 우 (MD370.83, MD371.83, MD372.83) V/1983 (Fontenille).

Holotype mâle (fig. 4). Tête. Vertex orné d'écailles jaunes et marron sombre. Une large tache d'écailles jaunes et blanchâtres déborde largement de l'espace interoculaire. Une autre large tache bien marquée, formée d'écailles larges et jaunâtres, occupe l'aire postgénale. Pas d'écailles claires bordant l'œil entre ces 2 taches latérales et la tache interoculaire. Présence d'écailles fourchues noires. Proboscis entièrement recouvert d'écailles noires. Palpe maxillaire nettement plus long que le proboscis et recouvert d'écailles sombres; les segments 3 à 5 portent 2 séries divergentes de longues soies disposées sur un même plan ; elles se réunissent sur l'apex du segment 4. Antenne. Pédicelle nu et brun sombre; verticilles des flagellomères 1 à 12 de couleur brun clair. Thorax. Tégument brun foncé. Scutum. Présence de bandes d'écailles jaunes se détachant nettement sur un fond d'écailles marron sombre. Il est entouré d'une large bordure d'écailles étroites jaune d'or et parcouru par 2 bandes dorso-centrales d'écailles falciformes jaunes qui se rejoignent peu avant l'aire préscutellaire. Cette aire est largement couverte d'écailles jaunes mais il subsiste une petite zone glabre médiopostérieure. Le reste du scutum est recouvert d'écailles marron foncé. Quelques soies dorso-centrales et acrosticales. Scutellum. Lobe médian couvert d'écailles jaune d'or ; quelques écailles analogues sur les lobes latéraux ; présence de 5 ou 6 longues soies sur chaque lobe. Partie latérale du thorax. Pronotum antérieur (Ap) portant quelques rares écailles claires; PA dépourvue d'écailles mais portant 4 à 5 soies fortes; Ppn sans écailles mais avec de nombreuses et longues soies. Proépisternum (Ps) avec des écailles claires et de nombreuses soies fortes. Quelques rares écailles claires couchées SSc. Présence de 3 taches bien visibles d'écailles larges et claires; 2 taches d'écailles larges et claires se trouvent l'une sur le Mks et l'autre sur le Mam. Les écailles de $\mathrm{MScU}$ sont couchées alors que celles MScL sont dressées; la tache $\mathrm{AMsC}$ du mésanépiméron est formée d'écailles couchées. Soie mésanépimérale inférieure absente. Aile recouverte d'écailles sombres; alule bordée de soies courtes; une frange de longues soies sur UC. Haltère. Pédicelle jaune très pâle ; capitellum recouvert d'écailles noires. Pattes. Coxae et trochanters bruns clairs avec des écailles claires antérieures; pattes noires à l'exception des fémurs; fémurs I et II, blancs sur leur face postérieure; fémur III clair avec un liseré sombre qui parcourt sa partie dorsale et s'élargit à l'apex. Griffes des pattes I à III simples. Abdomen. Présence de nombreuses longues soies dorsales et ventrales. Tergites I à VII recouverts d'écailles noires et brun foncé qui forment des dessins peu nets. Sur les tergites VI et VII 2 petites taches d'écailles blanchâtres latérales et antérieures sont visibles dorsalement. Sternites. Segments I à III dépourvus d'écailles; segments IV à VII avec des écailles claires localisées d'abord sur la partie basale et antérieure puis occupant progressivement l'ensemble du tergite à l'exception d'un étroite bande distale qui reste glabre et sombre sur le segment VII. Genitalia. Sternite IX : 4 à 6 soies fines de taille moyenne (plus longues que les soies analogues de $N e$. circumluteolus mais moins longues de celles de $\mathrm{Ne}$. belleci). L'édéage et les paraproctes sont analogues à ceux de $\mathrm{Ne}$. belleci et $\mathrm{Ne}$. nigropterum. Gonocoxite renflé sur son bord basal et interne; ce bombement porte de nombreuses soies longues, fines et souples. La face ventrale et interne du gonocoxite ne porte pas de soies en forme de baguette. Face dorsale du gonocoxite recouverte d'écailles et de soies longues et fortes. Le triangle terminal du gonocoxite est environ une fois et demie plus long que large; il porte une douzaine de soies courtes qui s'espacent progressivement pour devenir rares au niveau de l'articulation du style. Lobe basal du gonocoxite avec 2 courtes soies spiniformes et une soie souple. Gonostyle long et fin ; il présente une petite "visière " dorsale et ventralement une forte protubérance triangulaire en « soc de charrue "; griffe du gonostyle, longue, droite et terminée en brosse.

Femelle (fig. 2). Aspect général analogue à celui du mâle, mais elle présente les variations qui suivent. Tête. Palpe maxillaire recouvert d'écailles noires et portant quelques rares écailles argentées à l'apex. Antenne. Pédicelle jaune orangé ; flagelle 
noir. Thorax. Dorsalement, le tégument du thorax est brun très foncé; il devient progressivement plus clair pour être jaune pâle ventralement, au niveau des coxae. Antépronotum (Ap) recouvert d'écailles larges et sombres. Aire PA avec 2 ou 3 écailles claires et 4 à 5 soies postspiraculaires. Présence d'une tache d'écailles blanches sur SSc. Abdomen. En vue dorsale, l'abdomen apparaît entièrement sombre. Cependant, les tergites II à VII portent une tache d'écailles claires qui, devenant de plus en plus importante de l'avant vers l'arrière, apparaît en position latéro-antérieure sur les segments V à VII. Sternites I à VI recouverts d'écailles blanches; sternite VII plus sombre, présence d'écailles larges et claires à la base et sur les côtés. Cerque noire.

\section{Neomelaniconion fontenillei $\mathbf{n} . \mathbf{s p}$.}

Matériel type. La série type a été récoltée en janvier, février, février 1984, octobre 1986 et janvier 1987, dans la station forestière de Périnet/Andasibe [18 $8^{\circ} 55^{\prime} \mathrm{S} 48^{\circ} 25^{\prime} \mathrm{E}$ ] (Province de Toamasina), à une altitude voisine de $900 \mathrm{~m}$ (Fontenille). Les
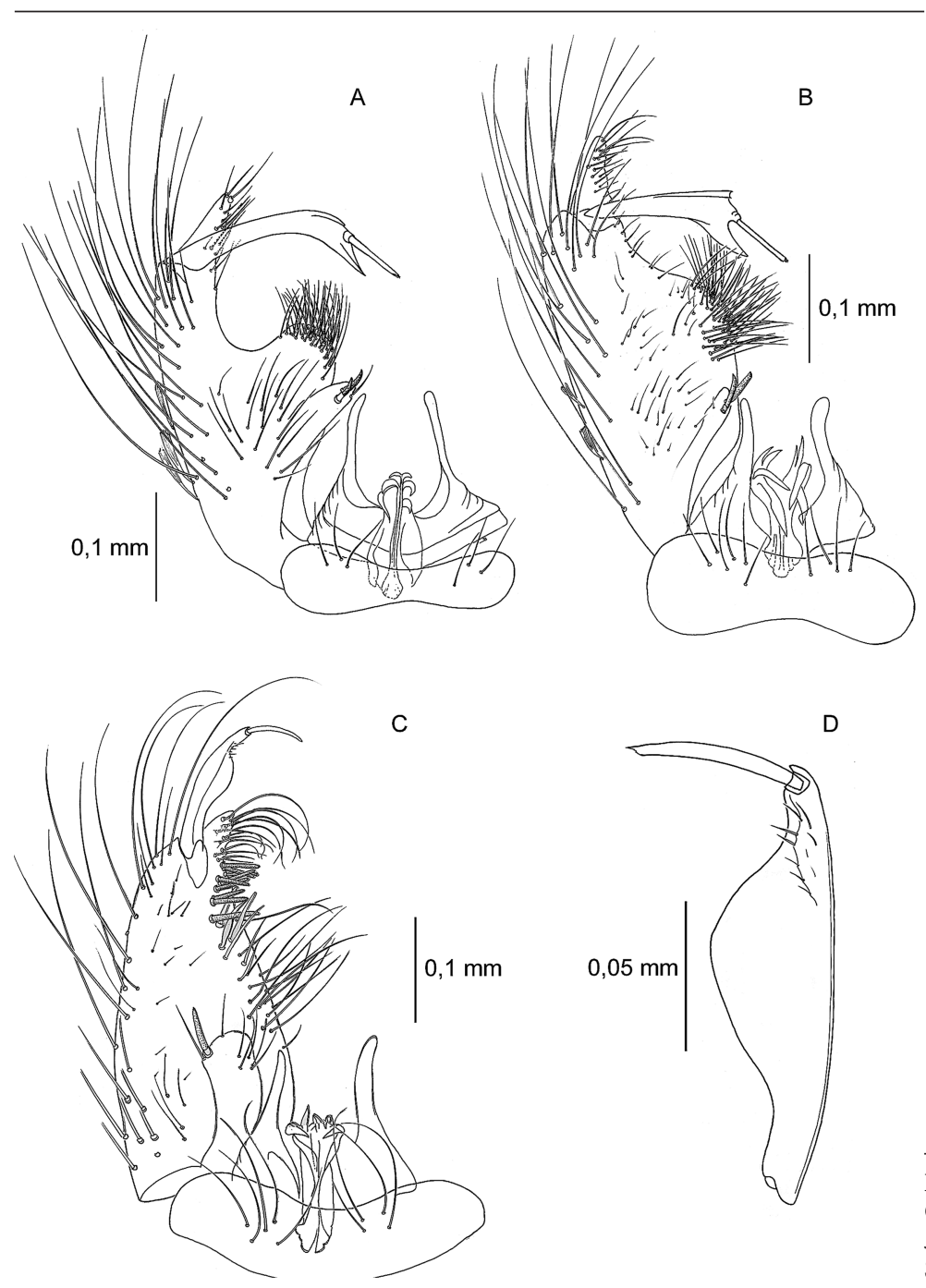

adultes ont été collectés en sous bois, sur homme ou au filet à

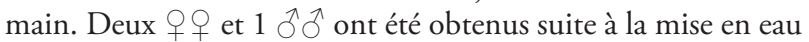
de boue sèche prélevée dans une petite dépression qui, en saison des pluies, devient une flaque. Les stades préimaginaux ont été perdus et sont donc inconnus.

Holotype : đótiqueté MD10.87, Périnet, novembre/1987. Paratypes : 2 औरे (MD6.86, MD30.86), 6/I/1986; 1 ते (MD97.86), 28/II/1986; 1 ते (MD231.86), 1/X/1986; 2

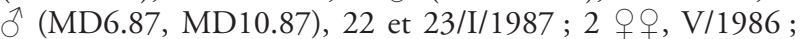
1 O (MD21.84), II/84; 1 र et 2 issus de l'élevage $n^{\circ} 7$, XI/87(Fontenille).

Dépôt des types. L'holotype $\hat{\jmath}, 4 \hat{\jmath} \widehat{\partial}$ (MD6.86, MD30.86, MD97.86, MD6.87) et 2 우 (V/1986 et MD97.86) seront conservés au Centre de Faunistique de l'IRD à Montpellier. Un đo (MD231.86) et 1 q (V/1986) seront conservés au Muséum national d'Histoire naturelle, Paris, France. Un $\widehat{\jmath}$ (MD10.87) et 1 ( $(\mathrm{MD} 21.84)$ seront déposés au Natural History Museum, Londres, Royaume-Uni.

Derivatio nominis. Espèce dédiée à Didier Fontenille qui a récolté cette espèce et qui l'avait reconnue comme nouvelle pour la science. Elle lui est aussi dédiée en hommage à ses travaux sur les Culicidae malgaches et à son soutien à la taxonomie.

Holotype mâle (fig. 5). Tête. Vertex brun foncé recouvert, dans l'espace interoculaire, d'écailles couchées étroites de couleur jaune d'or. Présence d'écailles couchées larges et blanchâtres sur l'aire postgénale (PG). Ecailles fourchues dressées et brunes sur l'occiput. Proboscis entièrement recouvert d'écailles noires. Palpe maxillaire nettement plus long que le proboscis et recouvert d'écailles sombres; segments 4 et 5 recourbés vers le haut; segment 5 très petit, triangulaire. L'apex du segment 3 et les segments 4 et 5 portent 2 rangées de soies ventrales divergentes qui se réunissent à l'apex du segment 4 . Antenne. Pédicelle nu et brun ; verticilles des flagellomères 1 à 12 bruns. Thorax. Tégument du scutum brun sombre devenant marron clair sur les côtés du thorax. Scutum bordé latéralement d'une large bande d'écailles jaune d'or et orné de 2 bandes dorso-centrales étroites d'écailles jaunes; aire préscutellaire recouverte d'écailles jaunes à l'exception d'une petite zone centrale et distale. Scutellum. Tégument brun; lobes latéraux portant 4 longues soies et quelques écailles falciformes jaune d'or; lobe médian recouvert d'écailles jaunes et portant 5 longues soies. Côté du thorax. Pronotum antérieur (Ap) parfois avec quelques rares écailles; Ppn glabre. Paratergite (PA) sans écailles et portant seulement 2 à 4 soies postspiraculaires. Ecailles SSc rares ou absentes. Proépisternum (Ps) orné de rares écailles blanchâtres et portant de nombreuses soies. Présence de 3 taches bien visibles formées

Figure 5

Genitalia.A, Neomelaniconionfontenillein.sp. ; B, Neomelaniconion sylvaticum n. sp.; C, Neomelaniconion nigropterum n. sp.; D, Style de Neomelaniconion nigropterum $\mathbf{n}$. sp. 
d'écailles larges et blanches aux reflets argentés : 2 taches se trouvent sur le Mks, la tache MScU est formée d'écailles couchées et la tache $\mathrm{MScL}$ est formée d'écailles dressées. La troisième tache se trouve sur le Mam en position antéro-supérieure. Soie mésépimérale inférieure absente. Aile. Entièrement recouverte d'écailles sombres; alule bordée de quelques courtes écailles ; une frange de longues soies sur UC. Haltère. Pédicelle clair; capitellum brun clair portant des écailles noires. Pattes. Coxae et trochanter jaune avec des écailles claires sur leur face antérieure ; pattes noires à l'exception des fémurs; fémurs I et II blancs sur leur face postérieure; fémur III clair avec un liseré sombre qui parcourt sa partie dorsale, en s'élargissant à l'apex. Griffes des pattes I à III simples. Abdomen. Présence de nombreuses et longues soies sur les tergites. Dorsalement, l'abdomen a un aspect brun. Tergites II à VII : bord distal sombre; le reste des segments est couvert d'un mélange d'écailles brunes et de rares écailles claires; les écailles claires sont plus nombreuses sur les V et VI ; elles sont nettement visibles en particulier sur les côtés du segment VII. Sternites brun clair recouverts d'écailles blanchâtres éparses, sternite VI plus sombre. Genitalia. Tergite IX avec de 3 à 4 longues soies souples. Gonocoxite. Présence d'une très forte expansion basale portant une grosse touffe de soies fines, souples et très serrées. Une zone glabre sépare cette expansion basale du niveau de l'insertion du gonostyle. Sur la face ventrale du gonocoxite, les soies sont peu nombreuses mais longues. Triangle terminal du gonocoxite avec de nombreuses soies courtes et non bouclées dont 1 ou 2 sont plus fortes que les autres ; bord externe de ce triangle terminal glabre. Gonostyle long et régulièrement arqué ; présence d'une visière dorsale peu marquée et d'une pointe triangulaire élancée entre lesquelles s'insère une baguette, longue, droite et terminée en brosse.

Femelle (fig. 2). Ornementation très semblable à celle du mâle. Tête. Taurus jaune et nu ; palpe très noir. Abdomen. Tergites uniformément sombres; sternites II à V couverts d'un mélange d'écailles jaunâtres et de quelques écailles sombres; sternites abdominaux VI et VII recouverts d'écailles blanches; sternite VIII et cerque noire.

Biologie. Ne. fontenillei n'a été jusqu'à présent récolté qu'à Périnet/Andasibe où les femelles sont agressives pendant la journée. Fontenille (com. pers.) a obtenu des larves, des nymphes et des adultes en mettant en eau de la terre sèche prélevée dans une petite dépression située en forêt. Malheureusement les stades préimaginaux ont été perdus et restent donc inconnus. Les œufs, résistants à la dessiccation, avaient été pondus avant l'assèchement de la petite mare temporaire et demeuraient prêts à éclore à la première mise en eau du gîte.

\section{Neomelaniconion albiradius $\mathbf{n} . \mathbf{s p}$.}

Matériel type. La série type a été récoltée en février 1987 dans la station forestière d'Ampijoroa [16 $13^{\circ} \mathrm{S} 46^{\circ} 28^{\prime} \mathrm{E}$ ] (Province de Mahajanga), à une altitude voisine de $100 \mathrm{~m}$ (Fontenille). Les 우 étaient agressives pour l'homme en sous-bois et pendant la journée. Les stades préimaginaux et les $\widehat{\partial} \widehat{\partial}$ sont, à ce jour, inconnus.

Holotype. $q$ étiquetée MD31.87, Ampijoroa, 14 février 1987. Paratypes : 1 (MD30.87), 14/II/1987; 1 ( $($ MD38.87), $15 /$ II/1987; 2 우 (MD42.87, MD43.87), 16/II/1987.

Dépôt des types. L'holotype $q$ ainsi que 2 paratypes $q+q$ seront déposés au Centre de faunistique de l'IRD à Montpellier. Un paratype $q$ (MD38.87), sera déposé au Muséum national d'Histoire naturelle, Paris, France et un autre (MD43.87) au Natural History Museum, Londres, Royaume-Uni.

Derivatio nominis. Nom en rapport avec la présence d'écailles blanches à la base de la nervure radiale.

Autre matériel étudié. Province d'Antananarivo. Relicte forestière, $40 \mathrm{~km}$ au sud de Tsiroanomandidy : $2+q$ agressives en début d'après-midi (MD35.85, MD39.85), 18/II1985, (Fontenille). Province de Mahajanga. Ampijoroa : $1 q$ agressive de jour, (MD126 83). Province de Toliara (Tuléar). Forêt dégradée proche de Mahabo (alt. $100 \mathrm{~m}$ ) : 4 q $q$ agressives le matin (MD293.86, MD294.86, MD295.86, MD296.86), 06/ XII/1986 (Fontenille).

Holotype femelle (fig. 2). Tête. Vertex de couleur brun foncé ; la partie médiane, autour de la suture coronale (cs), ainsi que l'espace interoculaire, sont recouverts d'écailles couchées étroites et jaune d'or ; de longues soies jaunes interoculaires sont aussi présentes. L'aire postgénale (PG) est recouverte d'écailles couchées blanchâtres. Ecailles fourchues noires sur l'occiput. Proboscis et palpe maxillaire entièrement recouverts d'écailles noires. Antenne. Pédicelle (Pe) orangé ; le premier flagellomère (FLm1) est jaune à la base et clair à l'apex. Thorax. Tégument brun sombre dans sa partie apicale (scutum) devenant progressivement plus clair sur les flancs du thorax pour être jaune pâle au niveau des coxae. Scutum brun sombre entouré d'une large bande d'écailles jaune d'or. Deux bandes dorso-centrales, très étroites, peu visibles, formées de l'alignement de quelques écailles jaune d'or. Ces bandes dorso-centrales deviennent plus larges au niveau de l'aire préscutellaire qu'elles entourent et recouvrent largement. Scutellum. Tégument sombre; des écailles jaune d'or sur les 3 lobes; 5 à 6 longues soies sur chaque lobe latéral et 4-5 soies disposées en éventail sur le lobe médian. Côté du thorax. Pronotum antérieur (Ap) orné au plus de quelques écailles claires; Ppn sans écailles ; Ap et Ppn sombres et portant de nombreuses et fortes soies noires. Proépisternum (Ps) recouvert d'écailles claires et portant de nombreuses soies. Sur PA sont implantées au moins 5 soies et des écailles claires. Bombement préalaire $(\mathrm{PK})$ noir avec de nombreuses soies sombres. Aire subspiraculaire (SA) orné d'écailles claires. Trois taches d'écailles larges et claires sont bien visibles : 2 d'entre elles se trouvent sur Mks; la tache MScU est formée d'écailles couchées alors que la tache MScL est formée d'écailles dressées. La troisième tache d'écailles claires se trouve sur le Mam en position supérieure (AMSc). Pas de soies mésanépimérales inférieures. Aile. Nervures couvertes d'écailles noires à l'exception du quart basal de la veine radiale $\mathrm{R}$ qui est orné d'écailles blanches. Haltère. Pédicelle blanchâtre; capitellum recouvert d'écailles noires. Pattes. Coxae et trochanters jaunes avec des écailles claires sur la partie antérieure. Fémurs et tibias I et II sombres sur la face antérieure et clairs sur la face postérieure. Tibia III sombre. Fémur III largement blanc: face antérieure ornée d'une bande sombre dorsale ; face postérieure claire sur sa moitié distale, sombre sur sa moitié apicale ; tarses sombres. Griffes des pattes I à III simples. Abdomen noir en vue dorsale. Tergites recouverts d'écailles noires; des écailles blanches sont cependant présentes sur les côtés des tergites II à VII ; ces écailles ne sont pas visibles dorsalement mais prolongent le revêtement blanc des sternites; cette bordure latérale d'écailles claires des tergites est parfois visible dorsalement sur les segments VI et VII. Sternites recouverts d'écailles blanches depuis le segment I jusqu'au segment VI ; sternite VII : clair à la base et portant des écailles sombres sur les 2/3 distaux. Cerque noire. 


\section{Clés d'identification}

\section{Femelles}

1. Aile avec des écailles blanches au moins sur la base de la radiale

- Aile avec seulement des écailles noires

4

2. Des écailles claires sur la radiale (R) et sur la médiane (M) ......................... Ne. circumluteolus

- Des écailles claires seulement sur la radiale .....

3

3. Tergites avec de nombreuses écailles claires éparses; quelques écailles claires sur l'extrême base de la radiale jusqu'au niveau de " $\mathrm{h} "$.................... Ne. Belleci n. sp.

- Tergites noirs. Des écailles blanches sur le 1/3 basal de la radiale .................................. Ne. albiradius n. $\mathbf{s p .}$

4. Scutum avec 2 bandes dorso-centrales nettes; bandes latérales entourant le scutum formées d'écailles jaune d'or .................................. Ne. sylvaticum n. sp.

- Scutum avec 2 bandes dorso-centrales à peine esquissées ; bandes latérales entourant le scutum formées d'écailles blanchâtres .

5

5. Des écailles sur l'aire postspiraculaire et sur l'aire subspiraculaire ....................... Ne. nigropterum n. sp. Pas d'écailles sur l'aire postspiraculaire, écailles rares ou absentes sur SA . Ne. fontenillei n. sp.

\section{Mâles}

Le mâle de $N e$. albiradius n'est pas connu.

1. Gonostyle renflé ventralement Groupe Circumluteolus Gonostyle non renflé ventralement Groupe Sylvaticum

2. Un seul type de soies en baguette sur la face ventrale et interne du gonocoxite; soies du tergite IX peu développées ................................. Ne. circumluteolus

- Deux types de soies en baguette sur la face ventrale et interne du gonocoxite; soies du tergite IX longues et fines

3. Gonostyle finement spiculé dorsalement ; nombreuses baguettes intermédiaires ................... Ne. belleci n. sp.

- Gonostyle portant une fine membrane apicale spiculée Ne. nigropterum n. sp.

4. Gonostyle avec une expansion ventrale en "soc de charrue "; lobe basal du gonocoxite peu marqué Ne. sylvaticum n. sp.

- Gonostyle sans expansion ventrale en "soc de charrue "; lobe basal du gonocoxite fortement marqué Ne. fontenillei n. sp.

\section{Discussion}

Alors que l'ornementation de l'ensemble des Neomelaniconion apparaît très homogène il est surprenant de distinguer plusieurs groupes d'architectures parmi les génitia des mâles. Plus de la moitié d'entre eux, soit 14 espèces, possède en effet un gonostyle fortement renflé ventralement (fig. 1, 4 et 5) ce qui confère une unité à ce groupe que nous proposons de nommer groupe Circumluteolus. Les membres de ce groupe ont souvent des bandes blanches basales sur les tergites abdominaux mais ce caractère ne saurait définir entièrement le groupe car 3 espèces, dont $N e$. belleci n. sp., ne le partagent pas. Il est à noter que la seule espèce orientale, $\mathrm{Ne}$. lineatopenne, appartient à ce groupe Circumluteolus; nous en discuterons la signification ci-dessous.

Ainsi défini, et compte tenu du fait que les mâles de certaines espèces ne sont pas connus, le groupe Circumluteolus comprend actuellement 14 espèces; il s'agit de : Ne. albicosta (Edwards 1913), Ne. albothorax, (Theobald 1907), Ne. aurovenatum (Worth 1960), $N e$. belleci n. sp., Ne. bequaerti (Wolfs 1947), Ne. bolense (Edwards 1936), Ne. circumluteolus (Theobald 1908), Ne. lineatopenne (Ludlow 1905), Ne. luridum (McIntosh 1971), Ne. luteolaterale (Theobald 1901), Ne. mcintoshi (Huang 1985), Ne. nigropterum n. sp., Ne. taeniarostris (Theobald 1909) et Ne. unidentatum (McIntosh 1971).

$N e$. belleci, dont le mâle présente des gonostyles renflés ventralement, appartient à ce groupe. Ses imagos se distinguent cependant des autres membres du groupe Circumluteolus par: l'absence, sur les tergites, de bandes claires basales, par la face dorsale de leur abdomen qui présente un mélange d'écailles claires et sombres ainsi que des spots blancs sur les angles antérieurs des tergites. Les écailles claires sont si nombreuses sur le tergite VII qu'elles le couvrent presque entièrement. Par ailleurs, les nervures alaires de $N e$. belleci ne présentent des écailles claires que sur la base de R. Cette nouvelle espèce se distingue aussi par ses pédicelles antennaires noirs et par l'ornementation de son scutum qui ne présente pas un contraste net entre les bandes latérales d'écailles jaunes et la partie centrale sombre. Les écailles jaunes sont simplement plus densément présentes sur les cotés du scutum que sur la partie centrale.

Les génitalias du mâle de $N e$ belleci sont très proches de celles de $\mathrm{Ne}$. circumluteolus dont elles se distinguent cependant par : un gonostyle délicatement pileux dorsalement; la présence sur le gonocoxite de 2 groupes de soies en forme de baguette. Le groupe distal est formé de baguettes courtes et sombres alors que le groupe basal est formé de baguettes plus longues et claires. Les soies fines et longues sont absentes au niveau de l'implantation des soies en baguette. Enfin, les soies portées par le tergite IX sont beaucoup plus longues que celles de Ne. circumluteolus.

Les larves du groupe Circumluteolus sont souvent méconnues et leur grande homogénéité a par ailleurs découragé leur représentation graphique tout comme 
leur description détaillée (Van Someren 1954 ; Huang 1985 ; McIntosh 1971). Le stade larvaire de Ne. aurovenatum a été cependant minutieusement décrit et dessiné par Cornel (Cornel 1991), celui de $N e$. taeniarostre par Bailly-Choumara (Bailly-Choumara 1965) et celui de Ne. bolense par Hamon et Rickenbach (Hamon \& Rickenbach 1954). En comparant ces larves à celle de Ne. belleci, il apparaît que les soies céphaliques de $N e$. belleci sont presque 2 fois plus longues que celle de $\mathrm{Ne}$. aurovenatum, de $\mathrm{Ne}$. taeniarostre et de $\mathrm{Ne}$. bolense. Par ailleurs, la soie 14-C est longue et double chez Ne. belleci alors qu'elle est courte et simple chez Ne. aurovenatum. Parmi les larves connues des espèces du groupe Circumluteolus, les principales originalités de celles de Ne. belleci nous semblent être : la soie 1-X très courte et très généralement double; les épines distales du siphon qui restent toujours en deçà de l'insertion de 1-S et qui présentent toujours une épine basale. Cette larve se distingue plus précisément de celle de $N e$. circumluteolus par sa soie 1-VIII qui ne possède que 5 à 6 branches courtes alors qu'elle est aussi développée que les soies 3 et 5 -VIII chez Ne. circumluteolus. Enfin, les dents terminales du peigne siphonal sont peu espacées chez Ne. belleci.

Les espèces $\mathrm{Ne}$. sylvaticum et $\mathrm{Ne}$. fontenillei, endémiques de Madagascar, n'appartiennent pas au groupe Circumluteolus. Leurs génitalias, dont le lobe basal du gonocoxite est à la fois très développé et pileux, leur confere immédiatement une forte originalité par rapport à tous les Neomelaniconion connus sur le continent africain (fig. 5). Labsence de soies en baguette ainsi que la forme de leur gonostyle confirment cette grande originalité. Il convient de les placer dans un groupe distinct que nous nommons groupe Sylvaticum.

Les autres espèces africaines qui appartiennent au genre Neomelaniconion possèdent des styles aux formes et aux ornementations variées qui ne permettent pas actuellement de déceler d'autres regroupements évidents.

Toutes les femelles du genre Neomelaniconion sont agressives pour l'homme pendant la journée. Cependant, si les populations sont importantes, l'agressivité peut se prolonger pendant la nuit.

Vingt sept des 28 Neomelaniconion actuellement connus ne sont présents que dans la région afro-tropicale. Parmi ces 27 espèces africaines, 4 ne se rencontrent qu'en Afrique de l'Ouest, 5 ont une large répartition qui va du Sénégal à la côte Est africaine ; 13 espèces n'ont été signalées que dans le Bassin du Congo ou en Afrique de l'Est et 5 sont endémiques de Madagascar. Une seule espèce: Ne. circumluteolus, dont l'aire de répartition est particulièrement vaste, a atteint les Comores et Madagascar.
Une seule espèce, $N$ e. lineatopenne (Ludlow 1905), est à la fois orientale et australienne mais n'appartient pas à la faune africaine (Huang 1985). Cette curieuse répartition souligne l'ancienneté du genre Neomelaniconion qui existait donc avant la séparation de l'Afrique et de la Région Orientale.

Le groupe Circumluteolus qui est présent en Afrique, à Madagascar et dans la région orientale, est probablement l'une des branches les plus anciennes du genre Neomelaniconion. Il est possible que ce groupe existait déjà au Crétacé Supérieur, il y a environ $70 \mathrm{M}$. A., au moment où les plaques africaine, malgache et orientale se sont séparées.

Les 6 espèces présentes dans la sous-région malgache, dont 5 sont endémiques, montrent une fois de plus que la radiation spécifique a été particulièrement active dans cette sous-région. Une telle observation confirme ce qui a été mis en évidence chez les Anopheles (Grjebine 1966), les Ficalbiini (Grjebine 1986), les Orthopodomyia (Brunhes \& Hervy 1995) et les Uranotaenia (Ramos \& Brunhes 2004).

\section{Références}

Bailly-Choumara H. 1965. Description de la larve et de la nymphe d'Aedes (Neomelaniconion) taeniarostris Theobald 1910. Observation sur une variation de coloration chez l'adulte. Bulletin de la Société de Pathologie Exotique 58: 671-676.

Brunhes J. 1977. Les moustiques de l'archipel des Comores I. Inventaire, répartition et description de quatre espèces ou sous-espèces nouvelles. Cahiers O.R.S.T.O.M., série Entomologie médicale et Parasitologie 15: 131-152.

Brunhes J., Hervy J.-P. 1995. Insectes Diptères Culicidae Culicinae Genre Orthopodomyia de la sous-région malgache et de la région afrotropicale. Faune de Madagascar, 85, ORSTOM-MNHN, Paris, 119 p.

Cornel A. J. 1991. Description of Aedes (Neomelaniconion) aurovenatus Worth (Diptera: Culicidae). Mosquito Systematics 23: 54-65.

Doucet J. 1951. Les moustiques de la région de Périnet. Mémoires de l'Institut Scientifique de Madagascar. Série (A) 6 (1): 63-82.

Edwards F.W. 1941. Mosquitoes of the Ethiopian Region. III. Culicine adults and pupae. British Museum (Natural History), Oxford University Press, London, $499 \mathrm{p}$.

Fontenille D. 1989. Etude des circuits de vection d'arbovirus, à Madagascar, 55, Archives de l'Institut Pasteur de Madagascar, Antananarivo, 317 p.

Grjebine A. 1966. Insectes Diptères Culicidae Anophelinae. Faune de Madagascar, 22, ORSTOM-CNRS, Paris, 487 p.

Grjebine A. 1986. Insectes Diptères Culicidae Culicinae Ficalbiini. Faune de Madagascar, 68, ORSTOM-MNHN, Paris, 441 p.

Hamon J., Rickenbach A. 1954. Contribution à l'étude des culicidés d'Afrique occidentale. Description d'Aedes (Aedimorphus) mattinglyi sp. n., Aedes (Banksinella) jamoti sp. n. Notes complémentaires sur Aedes (Aedimorphus) stokesi Evans, Aedes (Banksinella) bolensis Edwards. Bulletin de la Société de Pathologie Exotique 47: 930-941.

Harbach K. L., Knight K. L., 1980. Taxonomist' Glossary of Mosquito Anatomy. Department of Entomology, North Carolina State University, Raleigh, USA, 415 p.

Hopkins G. H. E. 1952. Mosquitoes of the Ethiopian Region. I. Larval bionomics of mosquitoes and taxonomy of culicine larvae. British Museum (Natural History), London, 355 p. 
Huang Y.-M. 1985. A new african species of Aedes (Diptera: Culicidae). Mosquito Systematics 17: 108-120.

Le Berre R., Hamon J. 1960. Description de la larve, de la nymphe et de la femelle d'Aedes (Neomelaniconion) jamoti Hamon et Rickenbach 1954, et révision des clés de détermination concernant le sous-genre Neomelaniconion en Afrique au sud du Sahara. Bulletin de la Société de Pathologie Exotique 53: 1054-1064.

McIntosh B.M. 1971. The aedine subgenus Neomelaniconion Newstead (Culicidae, Diptera) in southern Africa with descriptions of two new species. Journal of Entomological Society Southern Africa 34: 319-333.

Newstead R., Dutton J. E., Todd J. L. 1907. Insects and another Arthropoda collected in the Congo Free State. Annals of Tropical Medicine and Parasitology 1: 1-112.

Ramos H., Brunhes J. 2004. Insecta Diptera Culicidae Uranotaenia. Faune de Madagascar, 91, IRD-CIRAD-MNHN, Paris, 463 p.

Ravaonjanahary C. 1978. Les Aedes de Madagascar (Diptera, Culicidae). 1. Etude monographique du genre. 2. Biologie d'Aedes (Diceromyia) tiptoni. Travaux et Documents de l'O.R.S.T.O.M., 87, ORSTOM, Paris, 210 p.

Reinert J. F., Harbach R. E., Kitching I. J. 2004. Phylogeny and classification of Aedini (Diptera: Culicidae), based on morphological characters of all life stages. Zoological Journal of the Linnean Society 142: 289-368.

van Someren E. C. C. 1954. Ethiopian Culicidae: descriptions of a new Culex, the female of Eretmapodites tonsus Edwards and the early stages of two Aedes of the subgenus Banksinella Theobald. Proceedings of the Royal entomological Society of London 23: 119-126.

Wolfs J. 1947. Un culicide nouveau du Katanga, Aedes (Banksinella) bequaerti, sp. n. Revue de Zoologie et de Botanique Africaine 40: 40-41.

Worth C. B. 1960. Description of a new species of the Aedine subgenus Neomelaniconion from Tongoland, South Africa (Diptera: Culicidae). Journal of the Entomological Society Southern Africa 23: 312-313.
Annexe

Coordonnées des localités citées

\begin{tabular}{|c|c|c|c|}
\hline Localités & Provinces & Latitude E & Longitude $S$ \\
\hline Ambalavao & Fianarabtsoa & $21^{\circ} 52^{\prime}$ & $47^{\circ} 10^{\prime}$ \\
\hline Ambodimanga & Toamasina & $19^{\circ} 37^{\prime}$ & $48^{\circ} 16^{\prime}$ \\
\hline Ampijoroa & Mahajanga & $16^{\circ} 13^{\prime}$ & $46^{\circ} 28^{\prime}$ \\
\hline Andapa & Antsiranana & $14^{\circ} 13^{\prime}$ & $49^{\circ} 43^{\prime}$ \\
\hline Anjiro & Antananarivo & $18^{\circ} 53^{\prime}$ & $47^{\circ} 58^{\prime}$ \\
\hline Ankarafantsika & Mahajanga & $16^{\circ} 15^{\prime}$ & $46^{\circ} 55^{\prime}$ \\
\hline Dembeni & Mayotte & $12^{\circ} 45^{\prime}$ & $45^{\circ} 12^{\prime}$ \\
\hline Fenerive-Est & Toamasina & $17^{\circ} 23^{\prime}$ & $49^{\circ} 24$ \\
\hline Ifaho & Fianarantsoa & $22^{\circ} 10^{\prime}$ & $47^{\circ} 52^{\prime}$ \\
\hline Ile de Sainte Marie & Toamasina & $16^{\circ} 49^{\prime}$ & $49^{\circ} 55^{\prime}$ \\
\hline Ivato & Antananarivo & $18^{\circ} 48^{\prime}$ & $47^{\circ} 28^{\prime}$ \\
\hline Ivoloina & Toamasina & $18^{\circ} 05^{\prime}$ & $49^{\circ} 22^{\prime}$ \\
\hline La Mandraka & Antananarivo & $18^{\circ} 55^{\prime}$ & $47^{\circ} 55^{\prime}$ \\
\hline Lac Itasy & Antananarivo & $19^{\circ} 04^{\prime}$ & $46^{\circ} 47^{\prime}$ \\
\hline Lavatrandraka & Toamasina & $18^{\circ} 51^{\prime}$ & $48^{\circ} 04^{\prime}$ \\
\hline Maevatanana & Mahajanga & $16^{\circ} 57^{\prime}$ & $46^{\circ} 49^{\prime}$ \\
\hline Mahabo & Toliara & $24^{\circ} 22^{\prime}$ & $47^{\circ} 07$ \\
\hline Mampikony & Mahajanga & $16^{\circ} 02^{\prime}$ & $47^{\circ} 38^{\prime}$ \\
\hline Manja & Toliara & $21^{\circ} 25^{\prime}$ & $44^{\circ} 23^{\prime}$ \\
\hline Manombo & Fianarantsoa & $22^{\circ} 58^{\prime}$ & $47^{\circ} 47^{\prime}$ \\
\hline Marovitsika & Toamasina & $18^{\circ} 51^{\prime}$ & $48^{\circ} 04^{\prime}$ \\
\hline Marovoay & Mahajanga & $16^{\circ} 06^{\prime}$ & $46^{\circ} 37^{\prime}$ \\
\hline Moramanga & Toamasina & $18^{\circ} 55^{\prime}$ & $48^{\circ} 13^{\prime}$ \\
\hline Morondava & Toliara & $20^{\circ} 20^{\prime}$ & $44^{\circ} 16^{\prime}$ \\
\hline Nosy Be & Antsiranana & $13^{\circ} 19^{\prime}$ & $48^{\circ} 15^{\prime}$ \\
\hline Périnet/Andasibe & Toamasina & $18^{\circ} 55^{\prime}$ & $48^{\circ} 25^{\prime}$ \\
\hline Ranomafana & Fianarantsoa & $21^{\circ} 06^{\prime}$ & $47^{\circ} 21^{\prime}$ \\
\hline Sambava & Antsiranana & $14^{\circ} 16^{\prime}$ & $50^{\circ} 10^{\prime}$ \\
\hline Soalala & Mahajanga & $16^{\circ} 07^{\prime}$ & $45^{\circ} 21^{\prime}$ \\
\hline Tamatave & Toamasina & $18^{\circ} 10^{\prime}$ & $49^{\circ} 22^{\prime}$ \\
\hline Tananarive & Antananarivo & $18^{\circ} 55^{\prime}$ & $47^{\circ} 31^{\prime}$ \\
\hline Tsaramonenana & Antananarivo & $18^{\circ} 52^{\prime}$ & $45^{\circ} 59^{\prime}$ \\
\hline Tsiroanomandidy & Antananarivo & $18^{\circ} 46^{\prime}$ & $46^{\circ} 02^{\prime}$ \\
\hline
\end{tabular}

